\title{
On the upper critical field of the noncentrosymmetric superconductor BiPd
}

\author{
Darren C. Peets,, , 2, Ana Maldonado, ${ }^{1,3}$ Mostafa Enayat, ${ }^{1}$ Zhixiang Sun, ${ }^{1}$ Peter Wahl,,${ }^{1,3}$ and Andreas P. Schnyder ${ }^{1}$ \\ ${ }^{1}$ Max-Planck-Institut für Festkörperforschung, Stuttgart D-70569, Germany \\ ${ }^{2}$ Center for Correlated Electron Systems, Institute for Basic Science, \\ Seoul National University, Seoul 151-747, Korea \\ ${ }^{3}$ School of Physics and Astronomy, University of St. Andrews, \\ North Haugh, St. Andrews, Fife KY16 9SS, United Kingdom
}

(Dated: April 19, 2016)

\begin{abstract}
The superconducting parameters and upper critical field of the noncentrosymmetric superconductor $\mathrm{BiPd}$ have proven contentious. This material is of particular interest because it is a singular example of a $4 f$-electron-free noncentrosymmetric superconductor of which crystals may be grown and cleaved, enabling surface-sensitive spectroscopies. Here, using bulk probes augmented by tunnelling data on defects, we establish that the lower of the previously reported upper critical fields corresponds to the bulk transition. The material behaves as a nearly-weak-coupled BCS $s$-wave superconductor, and we report its superconducting parameters as drawn from the bulk upper critical field. Possible reasons behind the order-of-magnitude discrepancy in the reported upper critical fields are discussed.
\end{abstract}

PACS numbers: 74.25.Dw, 74.25.Op, 74.25.Bt, 74.70.Ad

\section{INTRODUCTION}

In noncentrosymmetric superconductors, the lack of spatial inversion symmetry means that the parity of the superconducting wavefunction is not a meaningful concept. Singlet and triplet pairing states are a consequence of parity, thus they are no longer eigenstates and can mix. With our usual simplifying assumptions for understanding superconductivity no longer valid, a vast array of exotic physics becomes possible $\stackrel{1,2}{2}$. Unfortunately, few such materials are known, many do not superconduct under ambient pressure, and single crystals have only been grown of a very few. In addition, significant spin-orbit splitting of the bands near the Fermi level is a required prerequsite for all proposed novel behaviour. As a consequence of these challenges, most theoretical predictions remain unrealized.

Known to be both superconducting ${ }^{3}$ and noncentrosymmetric $\underline{4}$ before $\mathrm{BCS}$ theory $\underline{5}^{\underline{5}}, \alpha$-BiPd was probably the first noncentrosymmetric superconductor identified as such. That noncentrosymmetric superconductors were particularly exotic is a far more recent discovery $\underline{\underline{6}}$, and this material is now attracting renewed attention, both as a noncentrosymmetric superconductor ${ }^{7-11}$ and for its topologically nontrivial surface states 12,13 . Several techniques have been applied to establish whether the material hosts novel physics arising from a mixed-parity condensate, including point-contact spectroscopy $\stackrel{14}{=}, \mathrm{NQR}^{15}$, and microwave susceptibility ${ }^{9}$, revealing tantalizing hints of such behaviour. The gap symmetry and pairing mechanism in this material, whether gap nodes occur, and the degree of parity mixing remain to be established. In fact, many of the material's most basic superconducting parameters remain hotly contested.

Of particular concern, reports of the material's upper critical field $H_{\mathrm{c} 2}$, from which fundamental supercon- ducting parameters such as the coherence length are extracted, vary by more than an order of magnitude. Recent resistivity and $a c$ susceptometry data suggest an upper critical field $H_{\mathrm{c} 2}$ around $0.8 \mathrm{~T}+\frac{7,8,14}{\text {, while more }}$ bulk-sensitive magnetization measurements and surfacesensitive scanning tunneling spectroscopy indicate a far lower value $\frac{12,16}{}$. Where the values are similar, the shape can disagree: $a c$ susceptometry $\underline{\underline{14}}$ leads to a very different $H-T$ phase diagram than that extracted from the resistive transition ${ }^{7}$. Here we present the bulk upper critical field $H_{\mathrm{c} 2}$ as determined by magnetization, resistivity and specific heat measurements, along with the superconducting parameters that may be extracted based on this upper critical field. Together with tunnelling data, these results paint a consistent picture of a singlet-dominated, fully-gapped pairing state, near weak-coupling BCS expectations. We discuss the likely reasons behind the discrepancy in previous reports.

\section{EXPERIMENTAL}

Crystals were grown by a modified BridgmanStockbarger technique, as described in greater detail elsewhere ${ }^{17}$. Chips of bismuth (Aldrich, 99.999\%) and palladium metal (Degussa or Credit Suisse, $99.95 \%$ ) in a stoichiometric ratio were sealed in an evacuated quartz tube with a conical end, which was cooled slowly through the material's congruent melting point $\left(600^{\circ} \mathrm{C} \underline{18,19}\right)$ in a temperature gradient, crystallizing the melt from one end of the tube at a growth rate of $1.5 \mathrm{~mm} / \mathrm{h}$. The ampoule was also cooled slowly through the transition between $\alpha$ - $\mathrm{BiPd}$ and $\beta$ - $\mathrm{BiPd} 20,21$ near $200^{\circ} \mathrm{C}$ to maximize the domain size. Reports thus far indicate that the material always exists as $\alpha$-BiPd below this transition, thus all measurements reported here were performed on the $\alpha$ phase, which we refer to simply as BiPd. The crystals 
were twinned and in many cases internally cracked due to the $\alpha-\beta$ phase transition, but otherwise single-domain.

Magnetization measurements were performed in Quantum Design MPMS-7 and MPMS-XL magnetometers with the RSO option, and $H_{\mathrm{c} 2}(T)$ was defined as the point where the sample reached $10 \%$ of its full, low- $H$, low- $T$ magnetization in zero-field-cooled measurements. Resistivity was measured in a Quantum Design PPMS by a standard four-wire technique on samples of approximate dimension $2 \times 0.5 \times 0.5 \mathrm{~mm}^{3}$, with a drive current of $5 \mathrm{~mA} ; H_{\mathrm{c} 2}(T)$ was defined as the midpoint of the transition. Note that there may be systematic errors in the resistivity due to the abovementioned cracking. To confirm the bulk, thermodynamic $T_{\mathrm{c}}$ and $H_{\mathrm{c} 2}$, specific heat was measured at low temperatures in a Quantum Design PPMS in zero field and for fields along the monoclinic $b$ axis. In this case, $H_{\mathrm{c} 2}(T)$ was found using an entropyconserving construction around the transition. Point defects were investigated using a home-built scanning tunnelling microscope (STM), operating in cryogenic vacuum at temperatures below $30 \mathrm{mK}^{22}$, on samples that were cleaved in situ at low temperatures.

\section{RESULTS}
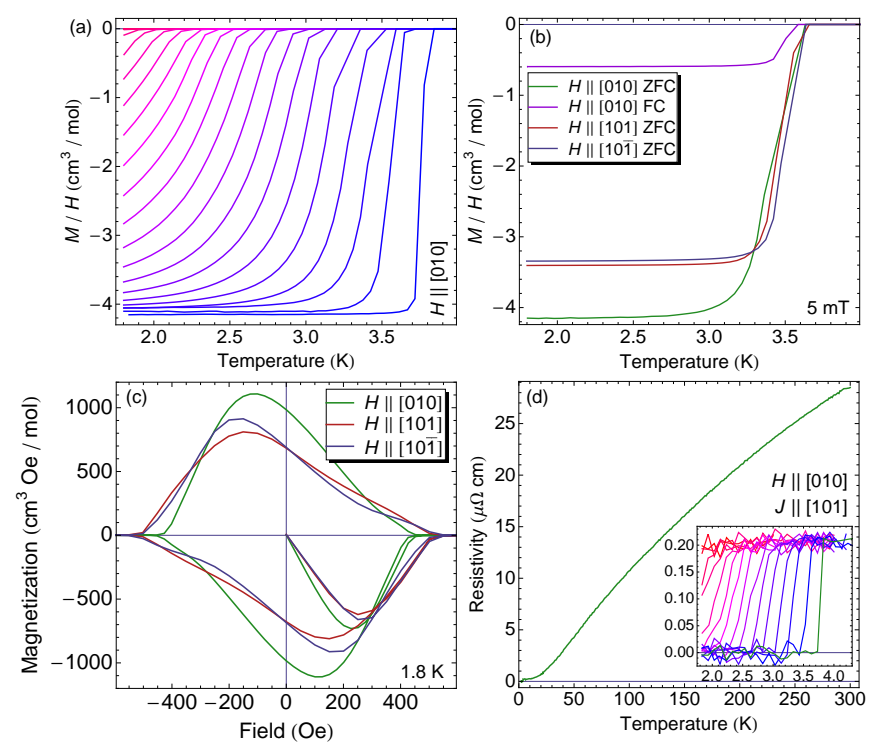

FIG. 1. (color online) Superconductivity in BiPd. (a) Zerofield-cooled magnetization curves in a field $H \|$ [010] of $0.1 \mathrm{mT}$, then every $2.5 \mathrm{mT}$ from 2.5 to $60.0 \mathrm{mT}$ (b) Magnetization curves in $H=5 \mathrm{mT}$ for three field orientations; (c) $M-H$ loops at $1.8 \mathrm{~K}$ for the three field orientations; and (d) zero-field resistivity - the inset shows the effect of adding fields $H \|$ [010] in steps of $5 \mathrm{mT}$.

Magnetization in a $5 \mathrm{mT}$ applied field is shown as a function of temperature in Fig. 1(b) for several field orientations, zero-field-cooled magnetization in a variety of fields $H \|[010]$ is presented in Fig. 1(a), and $M(H)$ loops at $1.8 \mathrm{~K}$ are shown in Fig. 1(c). The low-field transitions and $M(H)$ loops are essentially identical to those reported elsewhere $8,10,16$. The material is relatively isotropic, with the hysteresis loops exhibiting only minor differences with field orientation. We note that a recent report claimed ferromagnetism in BiPd based on an $M_{-}$ $H$ just above $T_{\mathrm{C}} \underline{10}$ - in our measurements, including in $M-H$ loops for all three field orientations at the same measurement temperature used in that report, no such hysteresis is observed and the magnetization observed is several orders of magnitude weaker. This recent report is also inconsistent with all $M(T)$ data and with previously published $M-H$ loops $\frac{16}{6}$, where such a ferromagnetic signal would be a significant fraction of the superconducting signal and clearly visible. Resistivity is also presented in Fig. 1(d), but absolute values may be unreliable due to internal cracking resulting from the $\alpha-\beta$ phase transition. The residual resistivity ratio of 140 is comparable with other work ${ }^{7,10}$, as is the resistive $T_{\mathrm{c}}$ of $3.8 \mathrm{~K}$.

Specific heat was measured from 0.37 to $5 \mathrm{~K}$ in fields up to $1.5 \mathrm{~T}$, to determine the bulk superconducting transition and gain insight into the structure of the gap function; results are presented in Fig. 2. Describing the slight curvature visible in the normal-state heat capacity in Fig. 2(d) and isolating the electronic contribution $c_{e l}(T)$ required the addition of small $T^{5}$ and $T^{7}$ corrections to the $T^{3}$ phonon term. The zero-field data are close to the weak-coupling BCS expectation 23 , with a clear exponential onset implying a full gap and suggesting a relatively isotropic gap function. As pointed out in our earlier paper ${ }^{12}$, recalculating the BCS form with the entropy-conserving $T_{\mathrm{c}}$ and the gap extracted from STM leads to a much better fit, without the need to introduce anisotropy or additional gaps, but implying a small deviation from weak coupling. The jump height $\Delta c_{e l} / \gamma T_{\mathrm{c}}$ at $T_{\mathrm{c}}$ is 1.50 , slightly higher than the BCS expectation of 1.43 , and the $T_{\mathrm{c}}$ of $3.77 \mathrm{~K}$ agrees well with that determined from the magnetization and resistivity measurements. Magnetic field suppresses the transition, which is completely absent above $0.4 \mathrm{~K}$ in a field of only $0.07 \mathrm{~T}$. It is worth noting at this point that this agrees well with the data presented in Fig. 1, but implies an upper critical field more than an order of magnitude lower than in the majority of recent reports. Fig. 2(d), which depicts all specific heat data above the bulk transition $H_{\mathrm{c} 2}(T)$ for all fields, shows that there is no evidence for a second phase transition which would correspond to the previously reported transition.

The transitions obtained from magnetization, resistivity and specific heat measurements taken in a variety of applied magnetic fields may be combined into an $H-T$ phase diagram, shown in Fig. 3. A lowtemperature point from the closing of the gap detected by STM is included 12 . The slight upward curvature observed near $T_{\mathrm{c}}$ is common in multi-band systems, and indeed band structure calculations indicate more than ten bands crossing the Fermi leve1 ${ }^{12,13}$. The data do not permit a reliable extrapolation to $H_{\mathrm{c} 2}(0)$, but it is clearly 

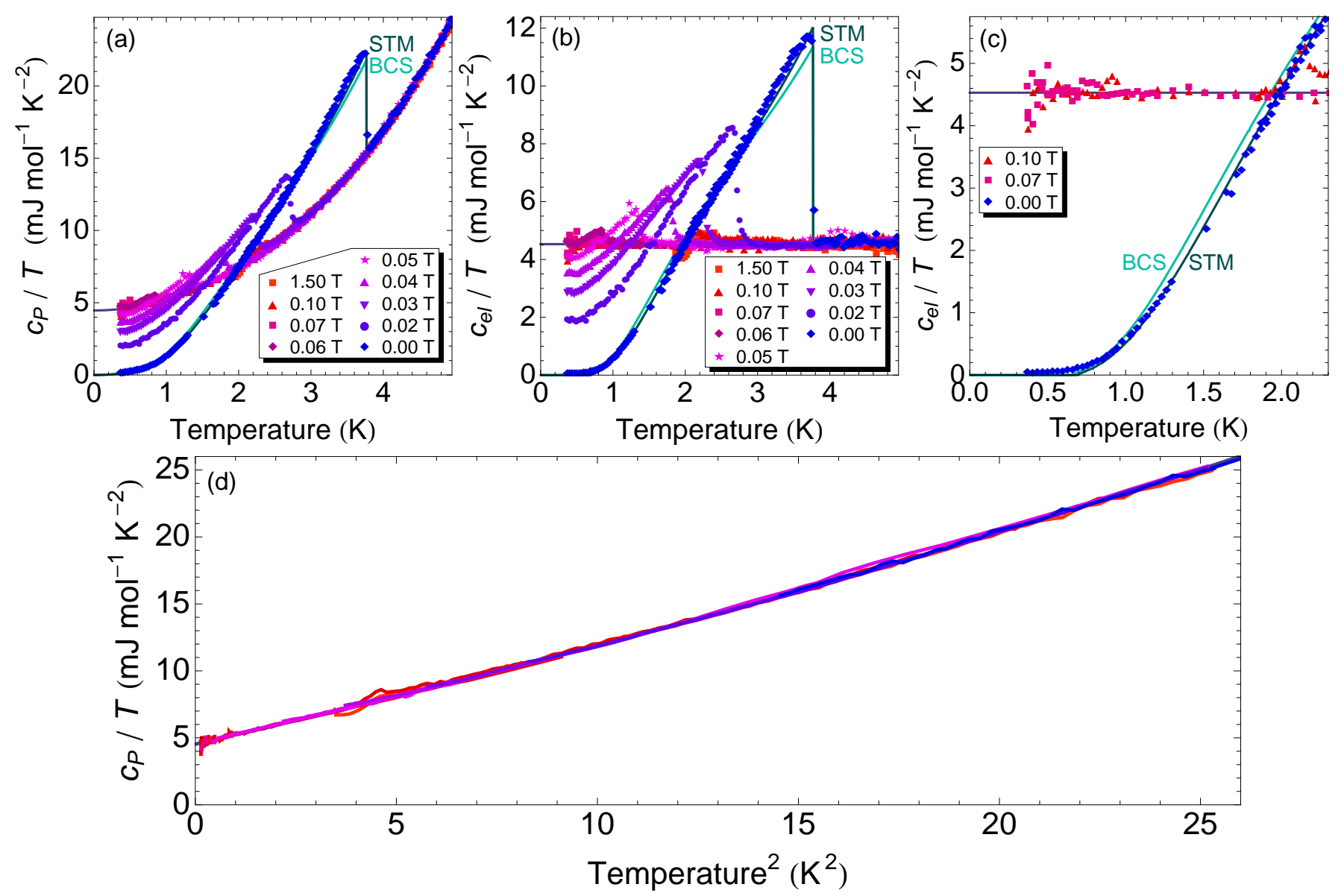

FIG. 2. (color online) Specific heat of BiPd. (a) $c_{P}(T) / T$ vs. $T$ for several fields, showing the bulk superconducting transition and its suppression by field. The weak-coupling $s$-wave BCS form ${ }^{23}$ and the BCS form recalculated using the STM gap 12 are included for comparison. There is no transition visible above $0.4 \mathrm{~K}$ in fields $H \geq 0.06 \mathrm{~T}$. (b) The electronic contribution $c_{e l}(T) / T$ vs. $T$. (c) The low-temperature region is fit better by the recalculated form based on the STM gap. (d) All data in fields of $70 \mathrm{mT}$ and above, and all data taken above the obvious (field-dependent) bulk transition, in all fields. There is no evidence to suggest an additional phase transition. The glitches near $4 \mathrm{~K}^{2}$ were not reproduced in later measurements.

the lower of the reported values. The discrepancy is discussed in greater detail below.

A variety of parameters characterizing BiPd and its superconductivity may be extracted from the specific heat. The Sommerfeld electronic specific heat coefficient $\gamma$ is a modest $4.53 \mathrm{~mJ} / \mathrm{mol} \mathrm{K}^{2}$, and the phonon $T^{3}$ term's prefactor $\beta$ is $0.710 \mathrm{~mJ} / \mathrm{mol} \mathrm{K}^{4}$, corresponding to a Debye temperature of $176 \mathrm{~K}$. The phonon contribution's clear departure from $T^{3}$ behaviour even as low as $3 \mathrm{~K}$ suggests that at least one phonon mode is rather low in energy. The zero-field electronic specific heat $c_{e l}$ may be integrated to obtain the thermodynamic critical field $H_{c}$, using

$$
\frac{\mu_{0} H_{c}^{2}}{2}=-\frac{\gamma T_{\mathrm{c}}^{2}}{2}+\int_{0}^{T_{\mathrm{c}}} c_{e l} d T
$$

in appropriately chosen volume units. For $H \|[010]$, the resulting thermodynamic $H_{c}^{b}$ of $41 \mathrm{mT}$, combined with the $H_{c 2}^{b}(0)$ of roughly $75 \mathrm{mT}$, would indicate a $\kappa_{G L}^{a c} \approx 1.3$. This indicates weakly Type-II superconductivity, near but not within the regime where the intermediate mixed phase is possible. The $H-T$ phase diagram is remarkably isotropic for in-plane fields, so the $a c$-plane coherence length $\xi^{a c}$ can be assumed to be isotropic to a good first approximation, and the $\xi^{a c}(0)$ extracted from $H_{c 2}^{b}(0)$ is $67 \mathrm{~nm}$. If the penetration depth is similarly isotropic, $\lambda^{a c}(0)=85 \mathrm{~nm}$. Because of the lower $H_{\mathrm{c} 2}$, this penetration depth is very far from the value reported previously and assumed in calculating the microwave penetration $\operatorname{depth}^{\underline{9}}$.

It is also possible to produce rough estimates of superconducting parameters for in-plane field orientations. On the assumption that the factor of $\sim 1.2$ anisotropy in Fig. 3 continues to zero temperature, $H_{c 2}^{a c}(0)$ would be $89 \mathrm{mT}, \kappa_{G L}^{b}$ would take a slightly stronger Type-II value of $1.5, \xi^{b}(0)$ would be $56 \mathrm{~nm}$, and $\lambda^{b}(0)$ would be $103 \mathrm{~nm}$. Confirmation of these values will be necessary, either by extending measurements of $H_{\mathrm{c} 2}^{i}$ to low temperature, or through independent measurements of the coherence length and penetration depth, perhaps from 


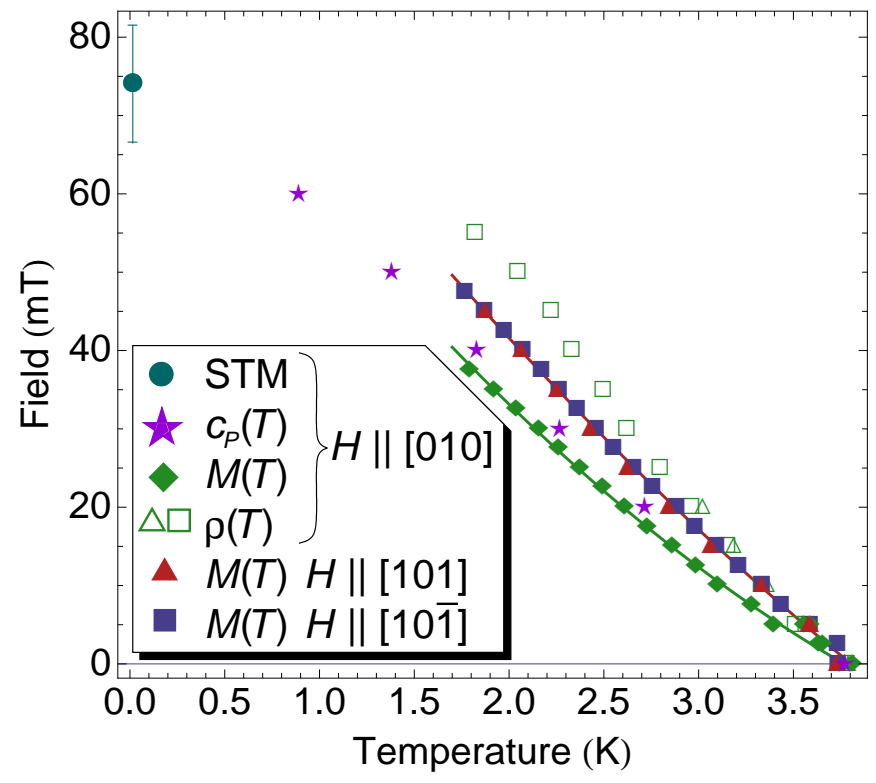

FIG. 3. (color online) $H-T$ phase diagram constructed from the transitions in the resistivity, magnetization and specific heat. A point corresponding to the closing of the gap as observed by $\mathrm{STM}^{12}$ is included.

muon spin rotation.

\section{DISCUSSION}

Upper critical fields on the order of $100 \mathrm{mT}$ have been reported based on magnetization measurements ${ }^{10,12,16}$, $\mathrm{STM}^{12}$, and now also specific heat; upper critical fields closer to $1 \mathrm{~T}$ have been inferred from resistivity ${ }^{7,8,10}$ and ac susceptometry ${ }^{14}$, although resistivity at high drive currents as reported here and in Ref. 12 appears to support the lower value, and an intermediate resistive value has also been reported ${ }^{11}$. Having concluded that the lower $H_{\mathrm{c} 2}$ represents the bulk, thermodynamic transition, the question arises as to the origin of the order of magnitude discrepancy. Possibilities include a significant difference in samples between the various groups, or some characteristic of the material that results in the survival of weak, either surface or filamentary, superconductivity above the bulk transition, which would short-circuit resistivity measurements.

The first consideration to raise here is whether Bi vacancies could alter the properties of the material. It has been established that BiPd can accommodate a considerable concentration of $\mathrm{Bi}$ vacancies, although not without degradation of the superconductivity ${ }^{8}$. However, this degradation is not rapid - the removal of a staggering $22 \%$ of all $\mathrm{Bi}$ atoms reduces the residual resistivity ratio by a factor of 50 but $T_{\mathrm{c}}$ only falls by $35 \%$. Perhaps more importantly, the resistive $H_{\mathrm{c} 2}$ appears to track the reduction in $T_{\mathrm{c}}$, making an order-of-magnitude jump unlikely. There are also no obvious features in the calcu- lated band structure ${ }^{12,13}$, such as a flat band, that would suggest fundamental changes to the carriers for carrier concentrations near the expected Fermi level. The picture emerging, again, is that of a relatively simple, BCS $s$-wave-like gap to first approximation. In our crystals, EDX analysis suggested a slight Bi deficiency, but it was within uncertainty of perfect stoichiometry. A more precise EPMA investigation was also consistent with ideal stoichiometry: $\mathrm{Bi}_{0.987(19)} \mathrm{Pd}_{1.013(18)}$ using $2 \sigma$ uncertainties. Apart from Ref. 8, in which all samples were far from stoichiometry, detailed information on the atomic ratios in the crystals studied is not available. However, the critical temperatures vary little among the remaining works, suggesting only minor deviations from ideal stoichiometry.
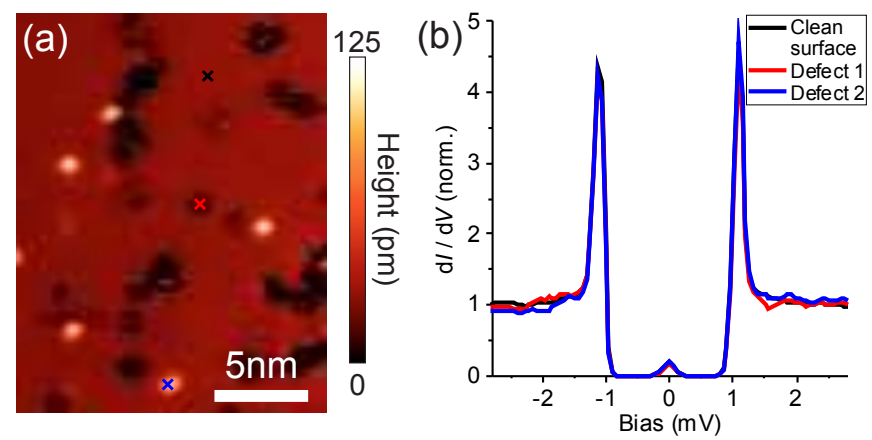

FIG. 4. (color online) Tunneling spectroscopy of individual defects. (a) Topographic image of defects in an atomically flat BiPd surface (settings: $V=50 \mathrm{mV}, I=0.1 \mathrm{nA}$ ). (b) Spectra taken on two different defects as well as the clean [010] surface, as marked in (a) with color-coded $\times$ signs (Defect 1 is dark). Spectra in panel (b) were obtained at $2 \mathrm{~K}$ using a superconducting tip with a BiPd cluster at its apex, resulting in coherence peaks at $\pm 2 \Delta$. The small peak at zero bias occurs at the difference between the tip and sample gaps due to the finite measurement temperature. An in-gap state would have produced an additional feature between the coherence peaks, which is not observed. The spectra on the defects show no obvious difference compared to the clean surface $(V=3.9 \mathrm{mV}$, $\left.I=2 \mathrm{nA}, V_{\mathrm{rms}}=70 \mu \mathrm{V}\right)$.

Having previously performed STM on these crystals 12 , we also had access to topographic and spectroscopic information on the observed point defects. Based on our topographical scans (e.g. Fig. 4a), point defects appear at approximately the $0.5 \%$ level per (surface) unit cell, or $0.25 \%$ per site.

Figure 4(b) compares tunneling spectra collected on two examples of point defects with spectra taken well away from them, using a superconducting tip. In the absence of any magnetic atoms, these defects can be expected to be non-magnetic scatterers. Comparisons of point defects with defect-free regions were performed at $15 \mathrm{mK}$ in an applied field of $35 \mathrm{mT}$ with a normal PtIr tip (not shown) and at $2 \mathrm{~K}$ in zero field with a superconducting BiPd tip. In both measurement modes, and on a range of distinct defects, spectra on defects were indistinguishable from those measured far from defects, and 
no in-gap states were observed. The small peak at zero bias voltage when using a superconducting tip arises due to the finite temperature of the experiment - tunnelling spectroscopy between two superconductors at finite temperature yields a peak at a bias corresponding to the difference of the two gaps, which increases in height on increasing temperature. Since our tip and sample are both BiPd, this peak appears at zero bias voltage. That point defects are indistinguishable from the bulk indicates that they aren't pair-breaking and are not harmful to the superconductivity. This is consistent with a full, non-sign-changing pairing gap (minor variations in phase would be possible).

Apart from point defects, the various samples could also differ in their concentration of extended defects, most obviously either inclusions or twin boundaries that occur at the $\alpha-\beta$ phase transition. Any such defects would introduce scattering, but we have already demonstrated that scattering does not have a strong effect on the superconductivity. $\beta-\mathrm{Bi}_{2} \mathrm{Pd}$ has a higher $H_{\mathrm{c} 2}{ }^{24,25}$, but also a higher $T_{\mathrm{c}}$ of $5.4 \mathrm{~K}$ that should be just as dominant in the zero-field resistivity as the higher apparent $H_{\mathrm{c} 2}$ transition is under applied field and low drive currents. Suppressing its $T_{\mathrm{c}}$ should also suppress its $H_{\mathrm{c} 2}$, making this an unlikely explanation. To the authors' knowledge, the low-temperature properties of $\mathrm{Bi}_{3} \mathrm{Pd}_{5}$ have not been reported. A wide variety of other $\mathrm{Bi}-\mathrm{Pd}$ phases exist $\frac{18,19}{19}$, but they should not be able to form as inclusions in a BiPd sample.

The crystals here were cooled slowly through the $\alpha-\beta$ structural transition, while other groups typically cooled rapidly through this temperature range, which should lead to significant differences in the concentration of twin boundaries and the spatial distribution of strain. The twin domain size could be smaller than the coherence length in some samples, perhaps making the material effectively centrosymmetric or leading to a reduced, effective coherence length. We cannot test this in other groups' crystals, but in STM work on our samples, the considerable difficulty in locating a twin boundary would strongly suggest a domain size well in excess of the zerotemperature coherence length, at least within the $a c$ plane. However, since the coherence length is strongly temperature-dependent, crystals with twin domains large compared to $\xi$ at low temperature would be in the opposite regime closer to $T_{\mathrm{c}}$. If any crossover existed between two regimes that differed by an order of magnitude in critical field, we would expect this crossover to be clearly visible. It is not.

The remaining possibility is that the bulk transition is masked from some measurement techniques - regions of many samples are clearly still superconducting well above the bulk $H_{\mathrm{c} 2}$. A surface critical field $\left(H_{c 3}\right)$ can explain a higher apparent transition in techniques sensitive to the sides of the sample where the applied field is parallel to the surface. In Ginzburg-Landau theory for a singlecomponent order parameter, $H_{c 3}=1.695 H_{\mathrm{c} 2}$, which would remain nearly an order of magnitude short of ex- plaining the discrepancy in upper critical fields. In noncentrosymmetric superconductors, however, the mixedparity condensate is not described by a single-component order parameter, and the constraints on $H_{c 3}$ would need to be rederived. Surface critical fields exceeding $1.695 \mathrm{H}_{\mathrm{c} 2}$ have been invoked in discussing discrepancies between resistivity and more bulk-sensitive probes in noncentrosymmetric $\mathrm{LaRhSi}_{3} \underline{26}, \mathrm{LaNiC}_{2} \underline{\underline{27}}, \mathrm{LaIrSi}_{3} \underline{28}$, and $\mathrm{LaPdSi}_{3} \underline{\underline{29}}$. However, a surface critical field cannot explain the thermal transport results on BiPd플 prised $\sim 30 \%$ of their cubic-millimeter-scale crystal.

The higher-field transition could also be filamentary, but the superconducting filaments must be sufficiently interconnected to permit lossless electrical transport, respond to $a c$ susceptometry, and prevent $\sim 30 \%$ of the carriers from participating in heat transport just above the bulk $H_{\mathrm{c} 2} 211$. The obvious network of extended defects throughout each sample is twin boundaries. Being twodimensional, these would support noticeable supercurrents, would be relatively well-connected throughout the sample, and would have enhanced apparent critical fields for fields oriented within the plane of the twin boundary, due to being in the thin limit. The loss of carriers in thermal transport could be explained by superconducting domain boundaries walling off areas of the sample or otherwise blockading the flow of heat - if $30 \%$ of the material were still superconducting, there should be clear signatures in the specific heat. Our $H_{\mathrm{c} 2}$ value would place the BiPd thermal transport data among the $s$-wave superconductors.

\section{CONCLUSION}

The data presented here paint a comprehensive picture of $\mathrm{BiPd}$ as a single-gap, nodeless, dominantly-swave BCS superconductor, albeit with slight deviations from weak coupling. The triplet component is apparently not strong enough to lead to significant gap anisotropy or nodes, as these would be seen in the specific heat. The upper critical field exhibits upward curvature suggestive of multi-band superconductivity, which is unsurprising given that band structure calculations show approximately 13 bands crossing the Fermi level ${ }^{12,13}$. Previously reported values of $H_{\mathrm{c} 2}$ disagree by more than an order of magnitude, and our results indicate that the lower values reflect the intrinsic behavior; superconducting parameters based on this are summarized above. Since parameters based on the higher $H_{\mathrm{c} 2}$ have been assumed in ensuing work, it would be desirable to recalculate some quantities, notably the microwave penetration $\operatorname{depth}^{9}$. The higher $H_{\mathrm{c} 2}$ values are most likely attributable to filamentary superconductivity occurring in thin regions along twin boundaries, although this remains to be demonstrated. As for why this would happen, strain is one candidate, but we note that isostatic pressure suppresses the superconductivity $\underline{\underline{10}}$. In $\mathrm{Sr}_{2} \mathrm{RuO}_{4}$, widely interpreted to be a chiral $p$-wave superconductor, the superconduct- 
ing onset temperature can double at boundaries with $\mathrm{Ru}$ metal inclusions ${ }^{30}$, and there is some evidence that this boundary may nucleate one component of the multicomponent order parameter $\stackrel{31}{1}$. The parity mixing in noncentrosymmetric superconductors implies that their condensates are effectively multi-component, so twin boundaries may exhibit analogous physics in BiPd.

\section{ACKNOWLEDGEMENTS}

The authors thank C. Stahl and E. Goering of Abt. Schütz, MPI-IS, for help with magnetization measure- ments, X. P. Shen for the EPMA measurements, and H. Jo for help measuring low-temperature specific heat. The authors are also grateful for the assistance of the MPI-FKF Crystal Growth and Chemical Service Groups. Work at IBS-CCES and SNU was supported by the Institute for Basic Science (IBS) in Korea (IBS-R009-G1), and work at St. Andrews was supported by the EPSRC through grant EP/I031014/1.

Data associated with this work are available in Ref. 32 .

* Current address: Advanced Materials Laboratory, Fudan University, Shanghai 200438, China; dpeets@fudan.edu.cn

${ }^{1}$ E. Bauer and M. Sigrist, eds.,

K. Kawashima, J. Akimitsu, and G.-Q. Zheng, J. Phys. Soc. Japan 82, 084711 (2013)

Non-Centrosymmetric Superondutors: B. Joshi, A. Thamizhavel, and S. Ra(Springer-Verlag, 2012).

2 S. Fujimoto, J. Phys. Soc. Japan 76, 051008 (2007)

arXiv:cond-mat/0702585 [cond-mat.supr-con]

3 N. E. Alekseevskii, Zh. Eksp. Teor. Fiz. 23, 484 (1952).

4 D. M. Kheiker, G. S. Zhdanov, and N. N. Zhuravlev, Zh. Eksp. Teor. Fiz. 25, 621 (1953).

5 J. Bardeen, L. N. Cooper, and J. R. Schrieffer, Phys. Rev. 106, 162 (1957).

6 E. Bauer, G. Hilscher, H. Michor, C. Paul, E. W. Scheidt, A. Gribanov, Y. Seropegin, H. Noël, M. Sigrist, and P. Rogl, Phys. Rev. Lett. 92, 027003 (2004)

7 B. Joshi, A. Thamizhavel, and S. Ramakrishnan, Phys. Rev. B 84, 064518 (2011).

8 K. Okawa, M. Kanou, T. Katagiri, H. Kashiwaya, S. Kashiwaya, and T. Sasagawa, Physics Procedia 45, 101 (2013)

9 L. Jiao, J. L. Zhang, Y. Chen, Z. F. Weng, Y. M. Shao, J. Y. Feng, X. Lu, B. Joshi, A. Thamizhavel, S. Ramakrishnan, and H. Q. Yuan, Phys. Rev. B 89, 060507(R) (2014), arXiv:1402.6154 [cond-mat.supr-con]

10 R. Jha, R. Goyal, P. Neha, V. Maurya, A. Srivastava, A. Gupta, S. Patnaik, and V. Awana, Supercond. Sci. Tech. 29, 025008 (2016), arXiv:1510.03217 [cond-mat.supr-con]

11 X. B. Yan, Y. Xu, L. P. He, J. K. Dong, H. B. Cho, D. C. Peets, J.-G. Park, and S. Y. Li, Supercond. Sci. Tech. 29, 065001 (2016)

12 Z. Sun, M. Enayat, A. Maldonado, C. Lithgow, E. Yelland, D. C. Peets, A. Yaresko, A. P. Schnyder, and P. Wahl, Nat. Commun. 6, 6633 (2015), arXiv:1407.5667 [cond-mat.supr-con]

13 M. Neupane, N. Alidoust, S.-Y. Xu, I. Belopolski, D. S. Sanchez, T.-R. Chang, H.-T. Jeng, H. Lin, A. Bansil, D. Kaczorowski, M. Z. Hasan, and T. Durakiewicz, "Discovery of the topological surface state in a noncentrosymmetric superconductor BiPd," (2015), arXiv:1505.03466 [cond-mat.mes-hall]].

14 M. Mondal, B. Joshi, S. Kumar, A. Kamlapure, S. C. Ganguli, A. Thamizhavel, S. S. Mandal, S. Ramakrishnan, and P. Raychaudhuri, Phys. Rev. B 86, 094520 (2012).

15 K. Matano, S. Maeda, H. Sawaoka, Y. Muro, T. Takabatake, B. Joshi, S. Ramakrishnan,

arXiv:1402.7232 [cond-mat.supr-con].

17 D. C. Peets, J. Phys.: Conf. Ser. 568, 022037 (2014).

18 B. Predel, "Bi-Pd (bismuth-palladium)," in Landolt-Börnstein - Group IV Physical Chemistry,

Vol. 5b, edited by O. Madelung (Springer-Verlag, 2014) Chap. 573, pp. 1-2.

19 J. Vřešt'ál, J. Pinkas, A. Watson, A. Scott, J. Houserová, and A. Kroupa, Calphad 30, 14 (2006).

${ }^{20}$ Y. Bhatt and K. Schubert, Journal of the Less Common Metals 64, 17 (1979)

21 V. M. Ionov, N. A. Tomlin, A. E. Prozorovskii, A. N. Klimenko, Y. V. Titov, S. G. Zhukov, and G. V. Fetisov, Sov. Phys. Crystallogr. 34, 496 (1989).

22 U. R. Singh, M. Enayat, S. C. White, and P. Wahl, Rev. Sci. Inst. 84, 013708 (2013).

23 B. Mühlschlegel, Zeitschrift für Physik 155, 313 (1959).

24 Y. Imai, F. Nabeshima, T. Yoshinaka, K. Miyatani, R. Kondo, S. Komiya, I. Tsukada, and A. Maeda, J. Phys. Soc. Japan 81, 113708 (2012), arXiv:1207.5905 [cond-mat.supr-con]

25 E. Herrera, I. Guillamón, J. A. Galvis, A. Correa, A. Fente, R. F. Luccas, F. J. Mompean, M. García-Hernández, S. Vieira, J. P. Brison, and H. Suderow, Phys. Rev. B 92, 054507 (2015), arXiv:1506.01411 [cond-mat.supr-con]

26 N. Kimura, N. Kabeya, K. Saitoh, K. Satoh, H. Ogi, K. Ohsaki, and H. Aoki, J. Phys. Soc. Japan 85, 024715 (2016)

27 Y. Hirose, T. Kishino, J. Sakaguchi, Y. Miura, F. Honda, T. Takeuchi, E. Yamamoto, Y. Haga, H. Harima, R. Settai, and Y. Ōnuki, J. Phys. Soc. Japan 81, 113703 (2012).

28 V. K. Anand, D. Britz, A. Bhattacharyya, D. T. Adroja, A. D. Hillier, A. M. Strydom, W. Kockelmann, B. D. Rainford, and K. A. McEwen, Phys. Rev. B 90, 014513 (2014).

29 M. Smidman, A. D. Hillier, D. T. Adroja, M. R. Lees, V. K. Anand, R. P. Singh, R. I. Smith, D. M. Paul, and G. Balakrishnan, Phys. Rev. B 89, 094509 (2014).

30 Y. Maeno, T. Ando, Y. Mori, E. Ohmichi, S. Ikeda, S. NishiZaki, and S. Nakatsuji, Phys. Rev. Lett. 81, 3765 (1998) 
31 T. Nakamura, R. Nakagawa, T. Yamagishi, T. Terashima, S. Yonezawa, M. Sigrist, and Y. Maeno, Phys. Rev. B 84, 060512(R) (2011) arXiv:1106.6091 [cond-mat.supr-con]
32 D. C. Peets, A. Maldonado, M. Enayat, Z. Sun, P. Wahl, and A. Schnyder, "Data underpinning - On the upper critical field of the noncentrosym (2016). 